\title{
Association between Variations in Body Mass Index and Cognitive Function in Older Korean Adults
}

\author{
Eun Mi Bae', Sang Min Park ${ }^{2,3, *}$ \\ 'Department of Family Medicine, National Police Hospital, Seoul,; ${ }^{2}$ Department of Family Medicine, Seoul National University Hospital, Seoul,; ${ }^{3}$ Department of \\ Biomedical Sciences, Seoul National University Graduate School, Seoul, Korea
}

Background: Although several studies have assessed obesity and cognitive impairment, most of these studies focus on body mass index (BMI) and cognitive impairment. Therefore to better understand the importance of weight maintenance with aging, this study investigated the relationship between variations in weight and cognitive impairment using the Korean version of the Mini-Mental State Examination (K-MMSE) in individuals aged 45 years or older in Korea.

Methods: Data on 3,477 adults with normal cognitive function (K-MMSE $\geq 24$ ) at baseline were acquired from the Korean Longitudinal Study of Aging (KLoSA) 2006-2016. The association between weight variability and risk of cognitive impairment was assessed using multiple logistic regression models. We also assessed weight variability and change in cognitive function over the 6-year follow-up using multiple linear regression.

Results: Overall, higher variations in BMI were associated with cognitive impairment. Patients in the quintile with the highest variation (Q5) in BMI (mean of BMI changes, 2.69) showed the greatest degree of cognitive impairments (adjusted odds ratio, 1.52; 95\% confidence interval [Cl], 1.08-2.14; $P$ for trend $=0.016$ ). Furthermore, a higher frequency in the number of times (3 times) the patient's body weight changed was associated with a lower cognitive function (adjusted odds ratio, 3.42; 95\% Cl, 1.67-7.03; $P$ for trend $<0.001$ ).

Conclusion: In this nationally representative study, weight variability was associated with a higher risk of cognitive decline during mid- and late-life stages.

Key words: Body weight change, Body mass index, Cognitive dysfunction, Dementia

Received April 7, 2021

Reviewed May 11, 202

Accepted May 22, 2021

*Corresponding author

Sang Min Park

https://orcid.org/0000-0002-7498-4829

Department of Family Medicine Seoul National University Hospital, 101 Daehak-ro, Jongno-gu, Seoul 03080, Korea

Tel: +82-2-2072-3331

Fax: +82-2-766-3276

E-mail: smpark.snuh@gmail.com

\section{INTRODUCTION}

Globally, obesity and an overweight health statuses have been associated with increased mortality and morbidity, and they are risk factors for cardiometabolic diseases, including diabetes mellitus, hypertension, cardiovascular disease, and some cancers. In a largescale, longitudinal study, high body mass index (BMI) was associated with increased risk of acute myocardial infarction and coronary heart disease even in young adults. ${ }^{1}$ Therefore, weight loss has been recommended as part of the efforts not only to reduce allcause mortality and morbidity, but also to improve the quality of life. Weight loss is usually followed by weight gain (weight cycling) and weight fluctuation, and this weight variability is an important risk factor for cardiovascular diseases and death. ${ }^{2,3}$ The importance of weight loss without weight variability has been emphasized in obese and overweight individuals. Despite the government's efforts to reduce the increasing prevalence of obesity and overweight statuses combined with weight cycling, the prevalence of these problems is steadily increasing.

As life expectancy increases, there is a corresponding and rapid increase in the number of cases of dementia worldwide. Cognitive impairment, dementia and Alzheimer diseases are known to impact the quality of later life. Adiposity is directly and indirectly associated with cognitive impairment and dementia. ${ }^{4}$ The relation- 
ship of BMI with the risk of dementia is consistent with a U-shaped curve, and mid-life obesity is a significant risk factor for dementia and Alzheimer disease. ${ }^{5}$ In contrast, some reports have insisted that overweight and obesity statuses serve as protective factors for cognitive impairment. ${ }^{6}$

The association between fluctuations in mid-life body weight and dementia is controversial. Being underweight or having a lower $\mathrm{BMI}$ in later-life is also associated with a poor prognosis for dementia compared to a stable BMI. ${ }^{7}$ Similarly, mid-life variations in body weight that persist for over 5 years are associated with an increased risk of dementia. ${ }^{8}$ Some studies have reported that high body weight variability was associated with an increased risk of dementia in the elderly. ${ }^{9,10}$ Other studies have reported that in women, additional weight gain or loss, in late life did not affect the risk of cognitive impairment, although this is not the case in men. ${ }^{11}$

In this study, we aimed to examine the association between weight variability and cognitive impairment in populations over 45 years of age. This study investigated changes in BMI and compared groups with healthy cognitive function and those with impaired cognitive function, using the Korean Longitudinal Study of Aging (KLoSA) database information from 2006 to 2016.

\section{METHODS}

\section{Data and study population}

The study population was acquired from the KLoSA, which is a nationally representative longitudinal survey of the population, 45 years of age and older, by the Korea Labor Institute and that has been collected since $2006 .{ }^{12}$ The participants were selected from a list of households that were comprised of at least one person aged 45 years or older in the census, based on a multistage stratified probability sampling scheme. The survey collected detailed information on health and cognitive function, demographics, income and assets, and employment on a biennial basis by conducting computer-assisted personal interviews. Currently, data from six waves (2006, 2008, 2010, 2012, 2014, and 2016) are publicly available; we used the first, second and third waves to assess weight variability and the first and sixth waves to assess cognitive changes. Of the 10,254 eligible individuals, for whom cognitive function was measured at baseline, 7,568 individuals with normal cognitive function (Korean

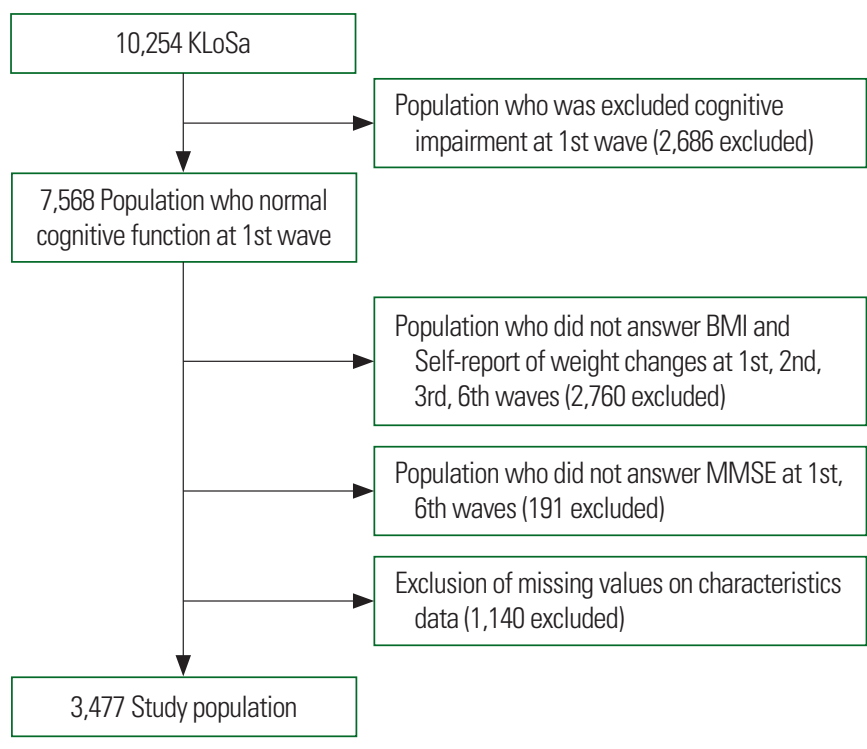

Figure 1. Diagram of final analytic sample using data from the Korean Longitudinal Study of Aging (KLoSA; 2006-20016). BMI, body mass index; MMSE, MiniMental State Examination.

version of the Mini-Mental State Examination [K-MMSE] score $\geq 24$ ) were defined as the baseline study population and 2,686 individuals were excluded because they were missing K-MMSE data in the first wave.

Among the 10,254 participants surveyed at baseline (2006), the following were excluded: 2,686 with mild or severe cognitive impairment (K-MMSE $\leq 23),{ }^{13}$ 2,760 with missing values for BMI and self-reported weight changes, 191 with missing values for MMSE scores, and 1,140 with missing values for characteristics data. Finally, a total of 3,477 participants remained in our sample for analysis (Fig. 1).

\section{Cognitive measures}

Cognitive performance was assessed using the MMSE, which is composed of seven categories representing different cognitive functions: orientation of time; orientation of place; registration of three words; attention and calculation; recall of three objects; language; and visual construction. ${ }^{14}$ This approach is a simple tool that is designed to assess the global cognitive status based on different cognitive domains and to help diagnose dementia. In general, a score of 23-18 is considered to be associated with cognitive impairment and $<18$ is regarded as severe cognitive impairment. This study assessed changes in cognitive function that were evaluated using the 
K-MMSE scores from 2006 to 2016.

\section{Weight variability}

Two methods were used to define weight variability: body-weight variability, and self-reported changes in weight. Measures of weight variability were assessed first, including the average successive variability (ASV). ASV was defined as individual variability in body weight between three visits. ASV in this study was defined as the absolute difference between successive values. ${ }^{15}$ The predictor variables were introduced as categorical variable (quintiles). ASV quintiles were defined as follows: Q1 was the quintile that had the least changes in BMI (mean, 0.20) and Q5 was the quintile that had the greatest changes in BMI (mean, 2.69). Self-reported weight changes were used as the second parameter for assessment. The predictor variables were calculated based on the questionnaire lists (from the 2006, 2010, and 2012 waves) and participants were asked whether there was a change of over $5 \mathrm{~kg}$ in body weight during the past year. The values from self-reported weight changes were used to assess self-awareness of weight changes.

\section{Covariates}

Additional covariates were collected from the 2006 survey. The socio-demographic variables included ages (45-54 years, 55-64 years, or > 65 years), sex (male or female), marital status (married or unmarried), education status (elementary school, middle school, high school, or college and higher), equalized household income quartiles, health insurance status (Medicaid or National Health Insurance), and location of residence (urban or rural). The analysis adjusted for health behaviors, such as smoking (ever or never), drinking (current or not current) and physical activity (regularly or not regularly). In addition, health status was assessed using the following variables: chronic disease status included hypertension, diabetes, cancer, chronic obstruction pulmonary disease, liver disease, cardiac disease, neurovascular disease, psychiatric illness, arthritis and prostate disease (none or one or more events) and depression symptoms (based on the Center for Epidemiological Studies Depression Scale: fewer than four or four or more symptoms).

\section{Statistical analysis}

To assess the association between weight variability and cognitive impairment, we evaluated cognitive decline on a continuous scale by using the change in the K-MMSE scored from 2006 to 2016. Multivariable logistic regressions were performed to assess cognitive impairments in 2016 (mild cognitive impairment and dementia), linear regressions were performed to assess the outcome of changes in the K-MMSE scores from 2006 to 2016 and estimated adjusted odds ratios (aORs). Multilevel analysis was performed to assess weight variability, based on measurements at three waves (2006, 2008, and 2010). We calculated ASV with BMI and the values provided in the self-reported weight changes. We selected normal K-MMSE scores at baseline to assess cognitive impairment and calculated the K-MMSE score changes in the 2016 wave.

For all analyses, Stata version 14 (StataCorp., College Station, TX, USA) was used, and the level of significance was set as 0.05 (two-sided). Longitudinal sampling BMI was employed and clustered standard error was estimated..$^{16}$ All participants were required to read and sign an agreement form before participating in the KLoSA study. The requirement for approval for this study for using human subjects was waived by the Institutional Review Board at the Seoul National University Hospital because this study used publicly available de-identified data.

\section{RESULTS}

\section{Descriptive statistics}

Table 1 shows demographic and clinical characteristics of the study participants at baseline. The average ( \pm standard deviation) baseline BMI was 23.43 ( \pm 0.04$)$, and there was no significant difference between the groups at baseline for BMI (Q1, 23.25 \pm 0.09 ; Q2, 23.45 0.09; Q3, 23.28 $\pm 0.10 ;$ Q4, 23.53 $\pm 0.09 ;$ Q5, $23.67 \pm$ 0.11 ). Across the age groups, the oldest group (aged $\geq 65$ years) had the largest proportion of the most BMI changes (Q5), and the fewest BMI changes (Q1) were identified in this population. Interestingly, the proportions of ASV differed by sex. The largest proportion of Q1 was identified in men, whereas that of Q5 was in women. Furthermore, the proportions of ASV differed significantly between lifestyles. Alcohol consumption (52.16\%) and physical activity $(47.13 \%)$ were associated with the lowest amounts of BMI change, whereas smoking was not associated with BMI change. 
Table 1. Descriptive characteristics of the study population

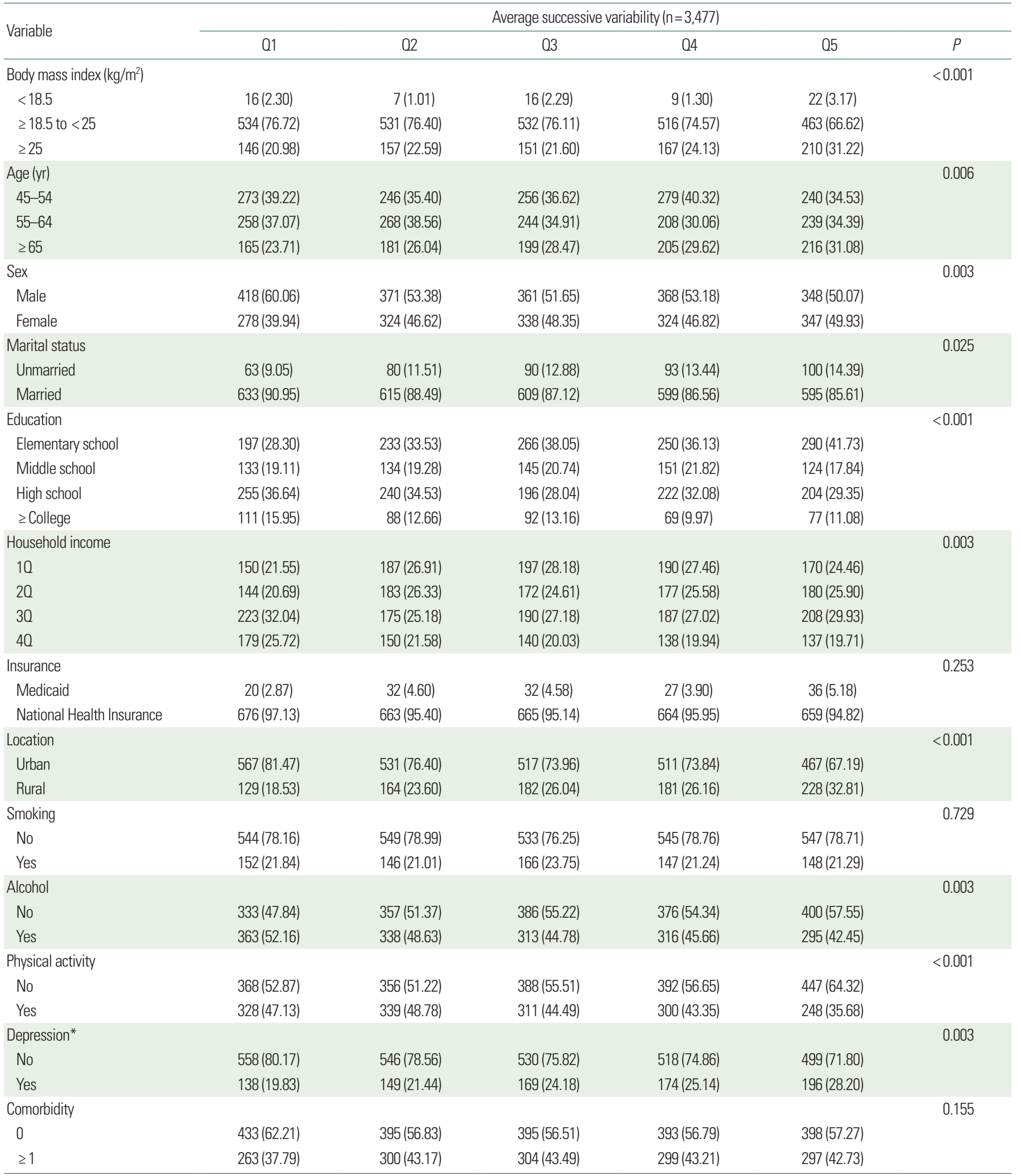

Values are presented as number (\%).

*The Center for Epidemiologic Studies-depression 10-item scale $\geq 4$ (yes), $<4$ (no). 
Table 2. Adjusted odds ratios of average successive variability for cognitive impairment in Korean adults $\geq 45$ years of age

\begin{tabular}{|c|c|c|c|c|c|c|}
\hline & 01 & 02 & 03 & 04 & 05 & Pfor trend \\
\hline Weight variability & $0.20 \pm 0.13$ & $0.56 \pm 0.09$ & $0.90 \pm 0.11$ & $1.42 \pm 0.20$ & $2.69 \pm 0.88$ & \\
\hline Crude & Ref & $1.45(1.06-2.00)$ & $1.58(1.16-2.16)$ & $1.67(1.22-2.28)$ & $1.97(1.45-2.68)$ & $<0.001$ \\
\hline Model $1^{*}$ & Ref & $1.32(0.94-1.86)$ & $1.41(1.00-2.00)$ & $1.48(1.05-2.08)$ & $1.56(1.11-2.20)$ & 0.010 \\
\hline Model $2^{\dagger}$ & Ref & $1.33(0.95-1.87)$ & $1.42(1.00-2.01)$ & $1.48(1.05-2.08)$ & $1.55(1.10-2.18)$ & 0.012 \\
\hline Model $3^{\ddagger}$ & Ref & $1.32(0.94-1.86)$ & $1.41(1.00-1.99)$ & $1.46(1.04-2.05)$ & $1.52(1.08-2.14)$ & 0.017 \\
\hline Model $4^{\S}$ & Ref & $1.32(0.94-1.86)$ & $1.41(1.00-1.99)$ & $1.46(1.04-2.05)$ & $1.52(1.08-2.14)$ & 0.016 \\
\hline Mild cognitive impairment ${ }^{\S}(17<$ K-MMSE $\leq 23)$ & Ref & $1.24(0.85-1.79)$ & $1.37(0.94-1.99)$ & $1.40(0.98-2.02)$ & $1.46(1.01-2.11)$ & 0.032 \\
\hline Severe cognitive impairment ${ }^{\S}$ (K-MMSE $\leq 17$ ) & Ref & $1.76(0.95-3.28)$ & $1.62(0.86-3.04)$ & $1.67(0.86-3.26)$ & $1.83(0.97-3.49)$ & 0.126 \\
\hline
\end{tabular}

Values are presented as mean \pm standard deviation or odds ratio ( $95 \%$ confidence interval).

${ }^{*}$ Model 1: adjusted for sex, age, marital status, education level, income, health insurance, living place; ${ }^{\dagger}$ Model 2: Model 1+smoking, alcohol, physical activity; ${ }^{\ddagger}$ Model 3: Model 2+comorbidity, depression; \$Model 4: Model 3+body mass index.

K-MMSE, Korean version of the Mini-Mental State Examination.

Table 3. Adjusted odds ratios for self-awareness of weight change values for cognitive impairment in Korean adults $\geq 45$ years of age

\begin{tabular}{|c|c|c|c|c|c|}
\hline & $0(n=2,024)$ & 1 Time $(n=1,006)$ & 2 Times $(n=367)$ & 3 Times $(n=80)$ & Pfor trend \\
\hline Crude & Ref & $1.30(1.05-1.62)$ & $2.07(1.55-2.78)$ & $3.09(1.76-5.43)$ & $<0.001$ \\
\hline Model $1^{*}$ & Ref & $1.17(0.92-1.48)$ & $2.06(1.48-2.85)$ & $3.56(1.74-7.24)$ & $<0.001$ \\
\hline Model $2^{\dagger}$ & Ref & $1.17(0.92-1.48)$ & $2.08(1.50-2.88)$ & $3.63(1.76-7.50)$ & $<0.001$ \\
\hline Model $3^{\ddagger}$ & Ref & $1.16(0.91-1.46)$ & $2.04(1.47-2.83)$ & $3.43(1.68-7.02)$ & $<0.001$ \\
\hline Model $4^{\S}$ & Ref & $1.15(0.91-1.46)$ & $2.06(1.49-2.86)$ & $3.42(1.67-7.03)$ & $<0.001$ \\
\hline Mild cognitive impairment ${ }^{\S}(17<$ K-MMSE $\leq 23)$ & Ref & $1.09(0.84-1.40)$ & $1.89(1.33-2.71)$ & $3.15(1.43-6.97)$ & $<0.001$ \\
\hline Severe cognitive impairment ${ }^{\S}(\mathrm{K}-\mathrm{MMSE} \leq 17)$ & Ref & $1.46(0.94-2.29)$ & $2.90(1.71-4.89)$ & $4.74(2.02-11.09)$ & $<0.001$ \\
\hline
\end{tabular}

Values are presented as odds ratio (95\% confidence interval).

${ }^{*}$ Model 1: adjusted for sex, age, marital status, education level, income, health insurance, living place; ${ }^{\dagger}$ Model 2: Model 1+smoking, alcohol, physical activity; ${ }^{\ddagger}$ Model 3: Model 2+comorbidity, depression; \$Model 4: Model 3+body mass index.

K-MMSE, Korean version of the Mini-Mental State Examination.

\section{Weight variability and cognitive impairment}

Table 2 presents the association between weight variability and overall cognitive impairment among all participants. Q1 was the quartile with the least amount of changes in BMI (mean, 0.20) and Q5 was the quintile with the greatest number of changes in BMI (mean, 2.69). Model 4 shows the estimates of aORs for Q2 (aOR, 1.32; 95\% confidence interval [CI], 0.94-1.86), Q3 (aOR, 1.41; 95\% CI, 1.00-1.99), Q4 (aOR, 1.46; 95\% CI, 1.04-2.05), and Q5 (aOR, 1.52; 95\% CI, 1.08-2.14) categories showed a linear trend in the risk of cognitive impairment $(P$ for trend $=0.016)$. A statistically significant association was observed between weight variability and development of mild cognitive impairment (K-MMSE $\leq 23$ ), although a significant association was not noted for severe cognitive impairment (K-MMSE $\leq 17)$.

The relationship between self-awareness of weight variability and cognitive impairment were also assessed, and then self-awareness of weight variability was assessed based on a self-administered questionnaire and cognitive function (Table 3). An increased frequency of changes in body weight was associated with mild cognitive impairment $(\mathrm{K}-\mathrm{MMSE} \leq 23)$ and severe cognitive impairment (K-MMSE $\leq 17)$ during the third follow-up. A linear trend was observed when the risk of mild cognitive impairment and severe cognitive impairment were assessed ( $P$ for trend $<0.001)$.

\section{Changes in cognitive function according to weight status}

Tables 4 and 5 presents the association between weight changes and cognitive function according to weight status. There was no statistically significant increase or decrease in final weight, but in the group that maintained their weight, weight variability was associated with cognitive impairment ( $P$ for trend $=0.02$ ). In comparison, self-awareness about weight change was significantly associated with cognitive impairment regardless of final weight $(P$ for trend $<0.001,0.023$, and 0.002). 
Table 4. Adjusted odds ratios for cognitive impairment of average successive variability

\begin{tabular}{|c|c|c|c|c|c|c|}
\hline & \multicolumn{6}{|c|}{ Odds ratio ( $95 \%$ confidence interval) of average successive variability* } \\
\hline & 01 & 02 & 03 & 04 & 05 & Pfor trend \\
\hline Decreased weight $(n=1,242)$ & Ref & $1.19(0.59-2.39)$ & $1.81(0.94-3.48)$ & $1.33(0.71-2.50)$ & $1.61(0.85-3.04)$ & 0.177 \\
\hline Stationed weight $(n=507)$ & Ref & $1.82(0.84-3.95)$ & $2.95(1.32-6.63)$ & $2.66(0.92-7.19)$ & $2.65(0.98-7.17)$ & 0.020 \\
\hline Increased weight $(n=1,728)$ & Ref & $1.28(0.82-2.03)$ & $0.97(0.61-1.56)$ & $1.32(0.92-2.10)$ & $1.33(0.83-2.13)$ & 0.260 \\
\hline
\end{tabular}

*Adjusted for sex, age, marital status, education level, income, health insurance, living place, smoking, alcohol, physical activity, comorbidity, depression, and body mass index.

Table 5. Adjusted odds ratios for cognitive impairment of self-aware about weight change number

\begin{tabular}{lccccc}
\hline & \multicolumn{4}{c}{ Odds ratio $(95 \%$ confidence interval) of self-aware about weight change number* } \\
\cline { 2 - 6 } & $0(\mathrm{n}=2,024)$ & 1 time $(\mathrm{n}=1,006)$ & 2 times $(\mathrm{n}=367)$ & 3 times $(\mathrm{n}=80)$ & $P$ for trend \\
\hline Decreased weight $(\mathrm{n}=1,242)$ & Ref & $0.90(0.56-1.43)$ & $2.63(1.45-4.77)$ & $8.50(2.53-28.48)$ & $<0.001$ \\
Stationed weight $(\mathrm{n}=507)$ & Ref & $2.25(1.21-4.15)$ & $1.61(0.60-4.33)$ & $2.87(0.71-11.62)$ & 0.023 \\
Increased weight $(n=1,728)$ & Ref & $1.14(0.83-1.57)$ & $1.91(1.23-2.96)$ & $2.16(0.96-4.87)$ & 0.002 \\
\hline
\end{tabular}

*Adjusted for sex, age, marital status, education level, income, health insurance, living place, smoking, alcohol, physical activity, comorbidity, depression, and body mass index.

\section{DISCUSSION}

The purpose of this study was to investigate the relationship between the extent of BMI change and cognitive impairment among a nationally representative sample of adults that were greater than 45 years of age. Our results suggested that large BMI changes were related to cognitive impairment. This relationship was also observed with the ASV of weight and self-awareness of the frequency of BMI changes.

Previous studies have found that obesity in mid-life is an important predictor of reduced cognitive performance, dementia, and Alzheimer disease in later life. ${ }^{1,17-19}$ Several studies have reported that weight loss precedes cognitive decline and impairment. ${ }^{20-22}$ However, there are only a limited number of studies that have assessed the relationship between changes in weight and cognitive function. Our results add new evidence which support that weight change, independent of baseline BMI, is significantly associated with the development of overall cognitive impairment. This finding has valuable implications for developing preventive strategies for cognitive impairment.

Although many studies have assessed weight change and cognitive function and suggested various mechanisms for the association, the relationship between the two is still unclear. Studies have investigated the relationship between the brain and obesity, which is related to the volume of gray and white matter. Obesity is sug- gested to be positively related to the process of increasing myelin and white matter volume. ${ }^{23}$ On the other hand, obesity is associated with decreased grey matter volume in the orbitofrontal cortex and right cerebellum. ${ }^{24}$

Other mechanisms include systemic inflammation and disruption of the metabolic system. Obesity is associated with systemic inflammation, which is associated with elevated levels of C-reactive protein, tumor necrosis factor, and serum amyloid A. These inflammatory markers affect cognitive impairment. ${ }^{25-27}$ Further, the insulin resistance observed in obese individuals is associated with cognitive function because insulin helps to regulate brain function and cognitive processes. Individuals with insulin resistance, such as those who are obese, are more likely to develop Alzheimer disease and individuals with Alzheimer disease are more likely to have increased plasma insulin. ${ }^{28,29}$

Additionally, BMI may have different effects based on age, sex, income groups, comorbidities and depression. ${ }^{30}$ Especially in agerelated studies, obesity has been noted to have a positive effect, whereas weight loss has a poor impact on cognitive function. ${ }^{20}$ Similarly, a systematic review has found that overweight individuals and those who are mildly obese are protected against mortality. ${ }^{31}$ These findings indicate that future research and interventions should critically assess these issues.

The present study had several limitations. First, because all weight measurements were self-reported, if overweight and obese adults 
systematically underreported their weight, the effect estimates in our study may be biased. However, this is unlikely because the selfreported anthropometric measures in the KLoSA subsample have previously been validated. ${ }^{32}$ Second, our findings on changes in the K-MMSE scores should be interpreted carefully in regard to factors, such as age and education, as well as the potential effects of measurement errors. It is not easy to determine how many changes in the K-MMSE scores occur normally, depending on age. Third, an unavoidable problem in longitudinal studies is selection bias due to attrition. Among 7,568 participants with normal cognitive function at baseline, about 53\% were excluded in the analysis due to the unavailability of weight information, missing K-MMSE scores and missing characteristic data. However, those excluded were similar to our analytic sample in respect to cognitive function. Fourth, this KLoSA database does not provide medication information or International Classification of Diseases codes, therefore, we were not able to identify medical conditions and could not account for the association between BMI weight fluctuations and dementia.

Despite the limitations, this study had several strengths. To our knowledge, this was the first study to explore the relationship between the ASV in body weight and cognition among an Asian population. We used a nationally representative sample that was followed longitudinally, which allowed us to prospectively measure and detect changes in the variables of interest. Moreover, data on a large number of potential confounders were available and allowed for appropriate adjustment in multivariate analyses.

In conclusion, our results demonstrate that a greater change in body weight was associated with cognitive impairment, regardless of baseline BMI. Previous studies with similar findings have recommended that older adults should consciously focus on maintaining bodyweight. ${ }^{33}$ Thus, based on the results of this study and previous analyses, we recommend minimizing weight changes or fluctuations throughout mid-life to minimize cognitive decline. Additional repeated longitudinal studies using representative samples are required to provide strong epidemiological evidence on the relationship between weight change and the risk of cognitive impairment.

\section{CONFLICTS OF INTEREST}

The authors declare no conflict of interest.

\section{ACKNOWLEDGMENTS}

The Korean Longitudinal Study of Aging is a public-use dataset, produced and distributed by the Korea Ministry of Employment and Labor and Korea Employment Information Service.

\section{AUTHOR CONTRIBUTIONS}

Study concept and design: EMB; acquisition of data: EMB; analysis and interpretation of data: EMB; drafting of the manuscript: EMB; critical revision of the manuscript: SMP; statistical analysis: EMB; administrative, technical, or material support: SMP; and study supervision: SMP.

\section{REFERENCES}

1. Choi S, Kim K, Kim SM, Lee G, Jeong SM, Park SY, et al. Association of obesity or weight change with coronary heart disease among young adults in South Korea. JAMA Intern Med 2018;178:1060-8.

2. Bangalore S, Fayyad R, Laskey R, DeMicco DA, Messerli FH, Waters DD. Body-weight fluctuations and outcomes in coronary disease. New Engl J Medicine 2017;376:1332-40.

3. Wannamethee SG, Shaper AG, Walker M. Weight change, weight fluctuation, and mortality. Arch Intern Med 2002;162:2575-80.

4. Luchsinger J, Mayeux R. Adiposity and Alzheimers disease. Curr Alzheimer Res 2007;4:127-34.

5. Gustafson D. Adiposity indices and dementia. Lancet Neurology 2006;5:713-20.

6. Smith E, Hay P, Campbell L, Trollor JN. A review of the association between obesity and cognitive function across the lifespan: implications for novel approaches to prevention and treatment. Obes Rev 2011;12:740-55.

7. Emmerzaal TL, Kiliaan AJ, Gustafson DR. 2003-2013: A decade of body mass index, Alzheimer's disease, and dementia. J Alzheimer's Dis 2015;43:739-55.

8. Ravona-Springer R, Schnaider-Beeri M, Goldbourt U. Body weight variability in midlife and risk for dementia in old age. Neurology 2013;80:1677-83.

9. Ha J, Kwon Y, Kwon Y, Kim D, Han K, Jang M, et al. Variabil- 
ity in body weight precedes diagnosis in dementia: A nationwide cohort study. Brain Behav 2020;10:e01811.

10. Roh E, Hwang SY, Kim JA, Lee YB, Hong S, Kim NH, et al. Body weight variability increases dementia risk among older adults: a nationwide population-based cohort study. Front Endocrinol 2020;11:291.

11. Noh HM, Han J, Kim YJ, Jung JH, Roh YK, Song HJ. Sex differences in the relationship between cognitive impairment and overweight or obesity in late life: a 3-year prospective study. Medicine 2019;98:e14736.

12. Korea Labor Institute. User guide for 2019 Korean longitudinal study of aging. Seoul: Korea Labor Institute; 2019.

13. Kang YW, Na DL, Hahn SH. A validity study on the Korean Mini-Mental State Examination (K-MMSE) in dementia patients. J Korean Neurol Assoc 1997;15:300-8.

14. Tombaugh TN, McIntyre NJ. The mini-mental state examination: a comprehensive review. J Am Geriatr Soc 1992;40:922-35.

15. Yeboah P, Hsu FC, Bertoni AG, Yeboah J. Body mass index, change in weight, body weight variability and outcomes in type 2 diabetes mellitus (from the ACCORD trial). Am J Cardiol 2019;123:576-81.

16. Korea Labor Institute. User guide for 2006 Korean longitudinal study of aging. Seoul: Korea Labor Institute; 2007.

17. Whitmer RA, Gunderson EP, Barrett-Connor E, Quesenberry $\mathrm{CP}$, Yaffe $\mathrm{K}$. Obesity in middle age and future risk of dementia: a 27 year longitudinal population based study. BMJ 2005;330: 1360.

18. Gustafson D. A life course of adiposity and dementia. Eur J Pharmacol 2008;585:163-75.

19. Lee SH, Han K, Cho H, Park YM, Kwon HS, Kang G, et al. Variability in metabolic parameters and risk of dementia: a nationwide population-based study. Alzheimer's Res Ther 2018; 10:110.

20. Bagger YZ, Tankó LB, Alexandersen P, Qin G, Christiansen C. The implications of body fat mass and fat distribution for cognitive function in elderly women. Obes Res 2004;12:1519-26.

21. Atti AR, Palmer K, Volpato S, Winblad B, Ronchi DD, Fratiglioni L. Late-life body mass index and dementia incidence: nine-year follow-up data from the Kungsholmen project. J Am Geriatr Soc 2008;56:111-6.
22. Stewart R, Masaki K, Xue QL, Peila R, Petrovitch H, White LR, et al. A 32-year prospective study of change in body weight and incident dementia: the Honolulu-Asia Aging Study. Arch Neurol 2005;62:55-60.

23. Taki Y, Kinomura S, Sato K, Inoue K, Goto R, Okada K, et al. Relationship between body mass index and gray matter volume in 1,428 healthy individuals. Obesity 2008;16:119-24.

24. Walther K, Birdsill AC, Glisky EL, Ryan L. Structural brain differences and cognitive functioning related to body mass index in older females. Hum Brain Mapp 2010;31:1052-64.

25. Reinehr T, Stoffel-Wagner B, Roth CL, Andler W. High-sensitive $\mathrm{C}$-reactive protein, tumor necrosis factor $\alpha$, and cardiovascular risk factors before and after weight loss in obese children. Metabolis 2005;54:1155-61.

26. Yang RZ, Lee MJ, Hu H, Pollin TI, Ryan AS, Nicklas BJ, et al. Acute-phase serum amyloid a: an inflammatory adipokine and potential link between obesity and its metabolic complications. PLoS Med 2006;3:e287.

27. Hung AM, Ikizler TA. Hemodialysis central venous catheters as a source of inflammation and its implications. Semin Dialysis 2008;21:401-4.

28. Razay G, Wilcock GK. Hyperinsulinaemia and Alzheimer's Disease. Age Ageing 1994;23:396-9.

29. Vanhanen M, Koivisto K, Kuusisto J, Mykkanen L, Helkala EL, Hanninen T, et al. Cognitive function in an elderly population with persistent impaired glucose tolerance. Diabetes Care 1998;21:398-402.

30. Prickett C, Brennan L, Stolwyk R. Examining the relationship between obesity and cognitive function: A systematic literature review. Obes Res Clin Pract 2015;9:93-113.

31. Flegal KM, Kit BK, Orpana H, Graubard BI. Association of all-cause mortality with overweight and obesity using standard body mass index categories: a systematic review and meta-analysis. JAMA 2013;309:71-82.

32. Yoon K, Jang SN, Chun H, Cho S. Self-reported anthropometric information cannot vouch for the accurate assessment of obesity prevalence in populations of middle-aged and older Korean individuals. Arch Gerontol Geriat 2014;59:584-92.

33. Brubacher D, Monsch AU, Stähelin HB. Weight change and cognitive performance. Int J Obesity 2004;28:1163-7. 